\title{
Cognitive Psychosocial Intervention in Dementia: A Systematic Review
}

\author{
Carme Carrion $^{a} \quad$ Marta Aymerich $^{a}$ Eva Baillés ${ }^{c}$ Abel López-Bermejo a, b \\ a TransLab Research Group, Department of Medical Sciences, School of Medicine, University \\ of Girona, and ${ }^{\mathrm{b}}$ Girona Institute for Biomedical Research, Girona, and ${ }^{\mathrm{c}}$ Departament de \\ Ciències Experimentals i de la Salut, Universitat Pompeu Fabra, Barcelona, Spain
}

\section{Key Words}

Dementia $\cdot$ Cognition-oriented therapies $\cdot$ Reality orientation $\cdot$ Skills training $\cdot$ Systematic review

\begin{abstract}
Background: The evolution of dementia depends on the underlying pathology, early diagnosis and the availability of effective treatment for some of the symptoms that interfere with the patients' or caregivers' quality of life. Even though there is no specific treatment to reverse dementia, some interventions such as reality orientation and skills training can retard cognitive impairment. Aim: To review existing scientific evidence regarding the efficacy of therapies included in the category of cognition-oriented approaches for people suffering from dementia. Methods: Papers were retrieved from several bibliographic databases (last publication date: 2009) with pre-specified selection criteria, data extraction and methodological quality assessment. Results: Nine reality orientation and 8 skills training trials were identified as meeting the inclusion criteria. Conclusions: Stimulation of cognitive functions, especially by means of reality orientation, improves overall cognitive function in patients suffering from dementia.

(C) 2013 S. Karger AG, Basel
\end{abstract}

\section{Introduction}

About $6.1 \%$ of the population aged 65 years and older suffer from dementia (about $0.5 \%$ of the worldwide population) and 59\% are female [1]. According to DSM-IV criteria, the essential feature of dementia is the development of multiple cognitive deficits that include memory impairment and at least one of the following cognitive disturbances: aphasia, apraxia, agnosia or a disturbance in execution abilities. This cognitive decline in dementia is usually 
accompanied by anxiety, depression and sleep disorders, and as anxiety and depressive symptoms increase, cognitive performance declines [2]. In addition, patients with dementia have at least three behavioural sub-syndromes: mood/apathy, psychosis or hyperactivity [3].

Alzheimer's disease is the most common form of dementia in Western countries (about half of all cases of dementia in elderly people). In 2006, the worldwide prevalence of Alzheimer's disease was 26.6 million, and by 2050, the prevalence will quadruple, by which time 1 in 85 people will be living with the disease worldwide [4]. The evolution of dementia depends on the underlying pathology, early diagnosis and the availability of effective treatment for some of the symptoms [5]. The degree of disability depends not only on the severity of the patient's cognitive impairment but also on the availability of social support. Like other neurodegenerative diseases, this disorder interferes with daily living activities and has important consequences not only for the patients' but also for the caregivers' quality of life $[6,7]$.

There are currently no treatments to reverse the course of dementia. However, certain treatments, both pharmacological and psychotherapeutic, do achieve a slowing of the impairment, especially with regard to cognitive deficits related to dementia. Patients with dementia exhibit a wide range of cognitive dysfunctions as well as behavioural and mood changes, and as a result often require an individualised and multimodal treatment plan. Essential to the treatment of patients with dementia is the psychiatric treatment of cognitive, functional and behavioural complications. The American Psychiatric Association (APA) [8] has described four different psychotherapeutic approaches that can be useful for treating people suffering from dementia: (1) cognition-oriented approaches (reality orientation, skills training); (2) emotion-oriented approaches (e.g. supportive psychotherapy, reminiscence therapy, validation therapy, sensory integration and simulated presence therapy); (3) behaviour-oriented approaches, and (4) stimulation-oriented approaches (e.g. recreational activities or therapies, music therapy, dance therapy, art therapy, exercise, multisensory stimulation and aromatherapy).

Although all of these treatments differ in philosophy, focus and methods, they have the broadly overlapping goals of improving function in the context of existing deficits. Cognitionoriented approaches aim to redress cognitive deficits; emotion-oriented approaches aim to maximize patients' mood; behaviour-oriented therapies aim to lessen or eliminate problem behaviours such as aggression or incontinence, and stimulation-oriented therapies include recreational and art therapies and aim to provide a stimulus that will help to decrease behavioural problems and improve mood. Cognition-oriented approaches seem to be the first to focus on as they aim to improve cognitive decline, the main sign of suffering from dementia.

The aim of this paper is to review existing scientific evidence on interventions included in the category of cognition-oriented approaches when treating people suffering from dementia. This category includes both reality orientation and skills training interventions.

\section{Methods}

Search Strategy

To identify the key studies, we used the following bibliographic databases: MEDLINE, EMBASE, PASCAL, The Cochrane Library (DARE, HTA Database, The Cochrane Database of Systematic Reviews, The Controlled Clinical Trials Database), The National Guidelines Clearinghouse, The UK Tripdatabase, Medline Plus, HEALTHSTAR, CINHAL and PSYCINFO. The bibliographic search was conducted in April 2010 and was restricted to English language papers or papers with an abstract in English. Additional papers were identified by searching the reference lists of the retrieved articles.

The search strategy was as follows: [dementia[majr] OR dementia [ti] OR Alzheimer[majr] OR Alzheimer[ti]] AND [psychotherapy[mh] OR psychotherapy[ti] OR cognitive therapy[mh] OR cognitive 


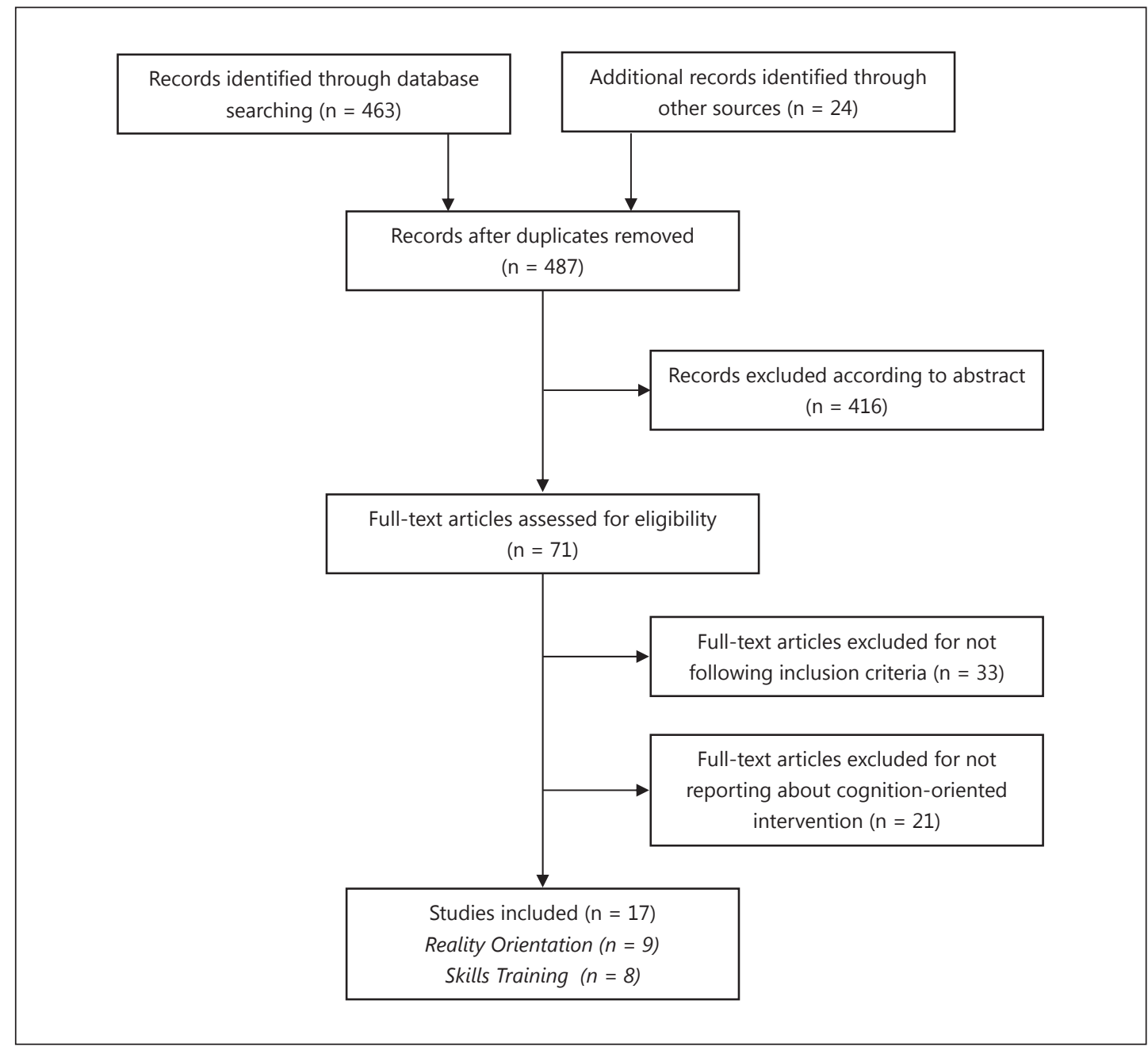

Fig. 1. Flow diagram of selection of papers for inclusion in the review.

therapy [ti]] and [randomized controlled trial[pt] OR controlled clinical trial[pt] OR clinical trial[pt] OR random*[ti] OR placebo*[ti] OR blind[ti] OR blinding[ti] OR trial* OR outcome* OR randomized controlled trials[mh] OR random allocation[mh] OR double blind method[mh] OR single blind method[mh] OR clinical trials[mh] OR placebos[mh] OR outcome assessment[mh] OR outcome*[ti] OR metaanalysis[pt] OR metaanal*[ti] OR meta-anal*[ti] OR systematic review [ti, ab] OR quantitative review[ti, ab] OR quantitative overview[ti, ab] OR systematic overview[ti, ab]].

Two reviewers independently screened the search results looking for eligible studies according to the content of their abstracts. Disagreements were resolved by consensus.

\section{Inclusion Criteria}

We selected articles that reported on intervention studies regarding cognition-oriented care approaches for dementia in older people diagnosed as having Alzheimer's disease or probable Alzheimer's disease. Only randomised controlled trials (RCTs) or controlled clinical trials were eligible.

\section{Data Extraction and Quality Assessment}

Data extraction was performed by two reviewers. Study features and outcomes were entered into a database specifically designed for this review. Quality criteria were based on SIGN checklists [9]. One reviewer assessed quality criteria and a second checked for accuracy. Disagreements were resolved by 


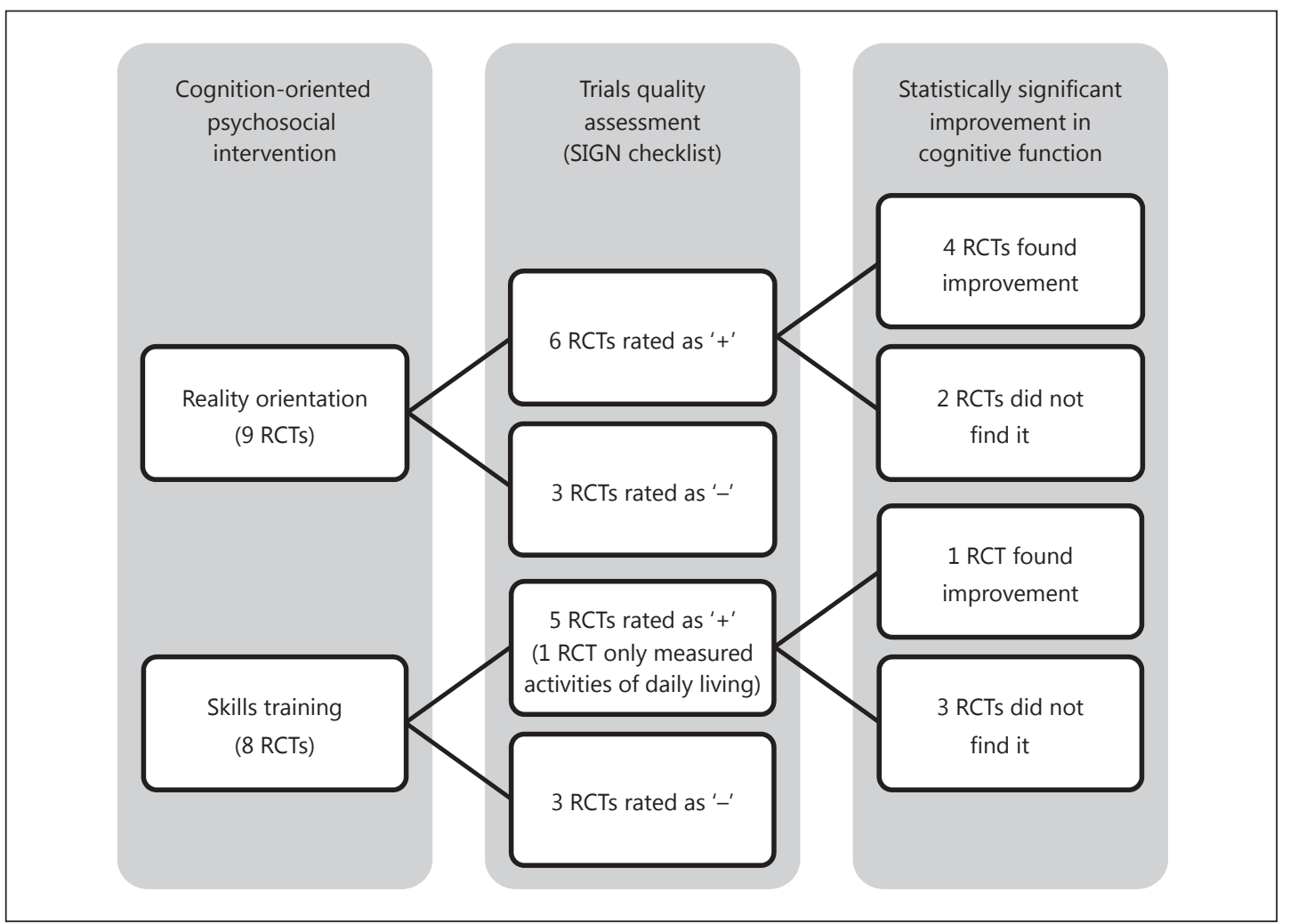

Fig. 2. Summary results of cognition-oriented approaches for dementia.

consensus and, if necessary, by a third reviewer. According to SIGN codes for study assessment, those trials that were clearly of an adequate quality were graded as '++' (RCTs with a very low risk of bias) or ' + ' (RCTs with a low risk of bias), while those of insufficient quality were graded as '-' (RCTs with a high risk of bias).

\section{Results}

The search process and total number of trials included in this review are illustrated in figure 1. An outline of the results is shown in figure 2, and details of all included trials are summarised in table 1 for reality orientation interventions and in table 2 for skills training interventions.

\section{Reality Orientation Interventions}

Reality orientation was first described in the second half of the 1960s as an aid to improve the quality of life of seniors with confusion [10]. This technique operates through the presentation and repetition of orientation information with the purpose of providing the patients with a better understanding of their surroundings. During the session, the therapist repeatedly presents basic personal and current information to each patient beginning with the patient's name, where he or she is and the date. When the patient has relearned these basic facts, others are presented such as age, hometown and former occupation. 
Dementia

Cognitive Disorders

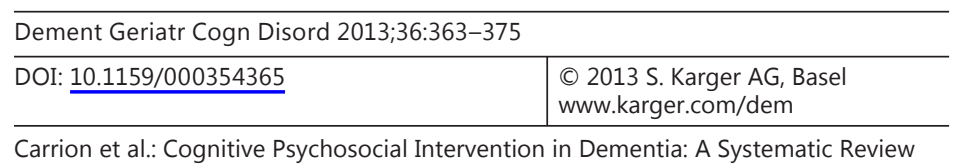

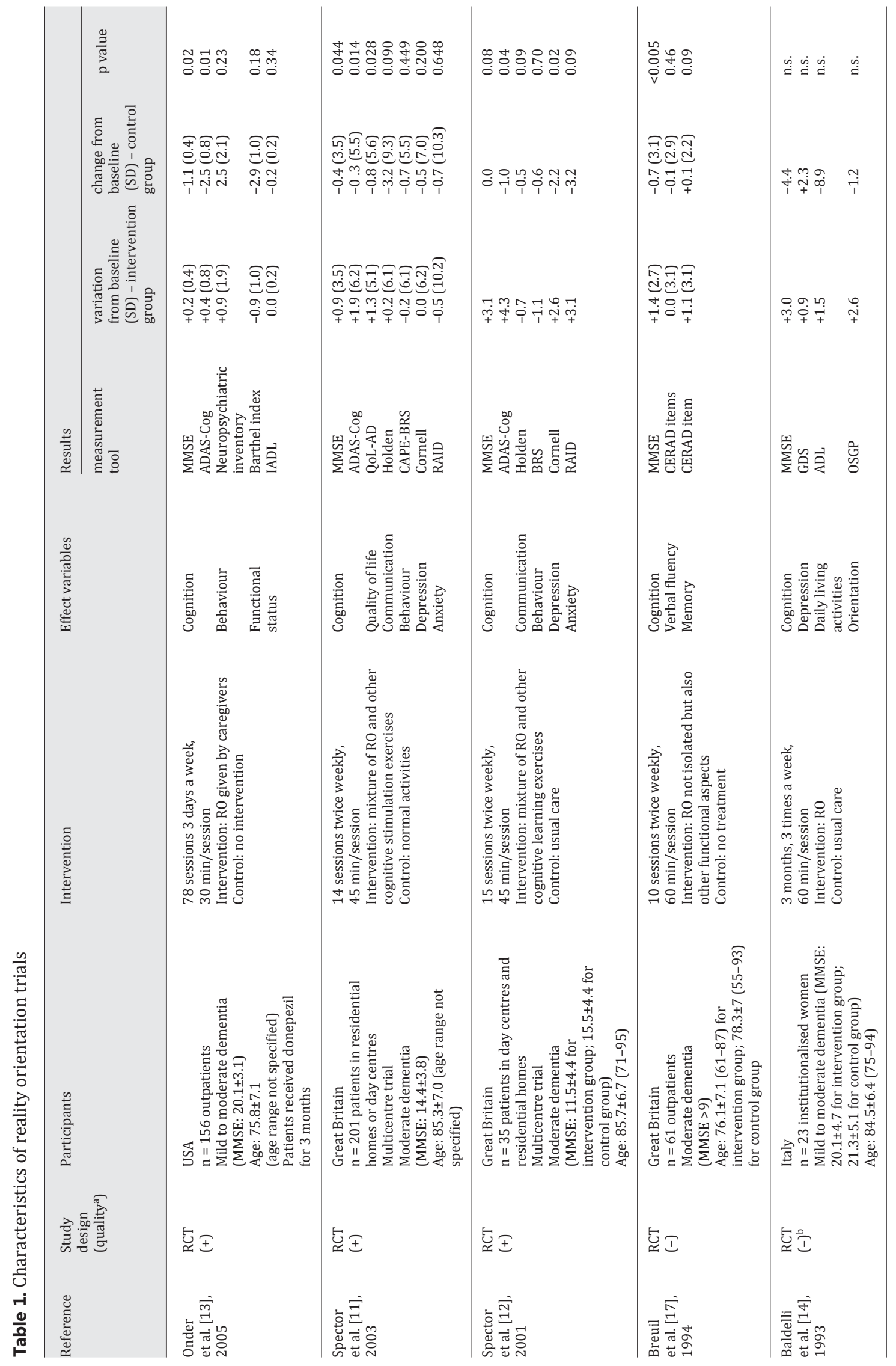


Dementia

and Geriatric
Cognitive Disorders
Dement Geriatr Cogn Disord 2013;36:363-375

DOI: $10.1159 / 000354365$

Carrion et al.: Cognitive Psychosocial Intervention in Dementia: A Systematic Review

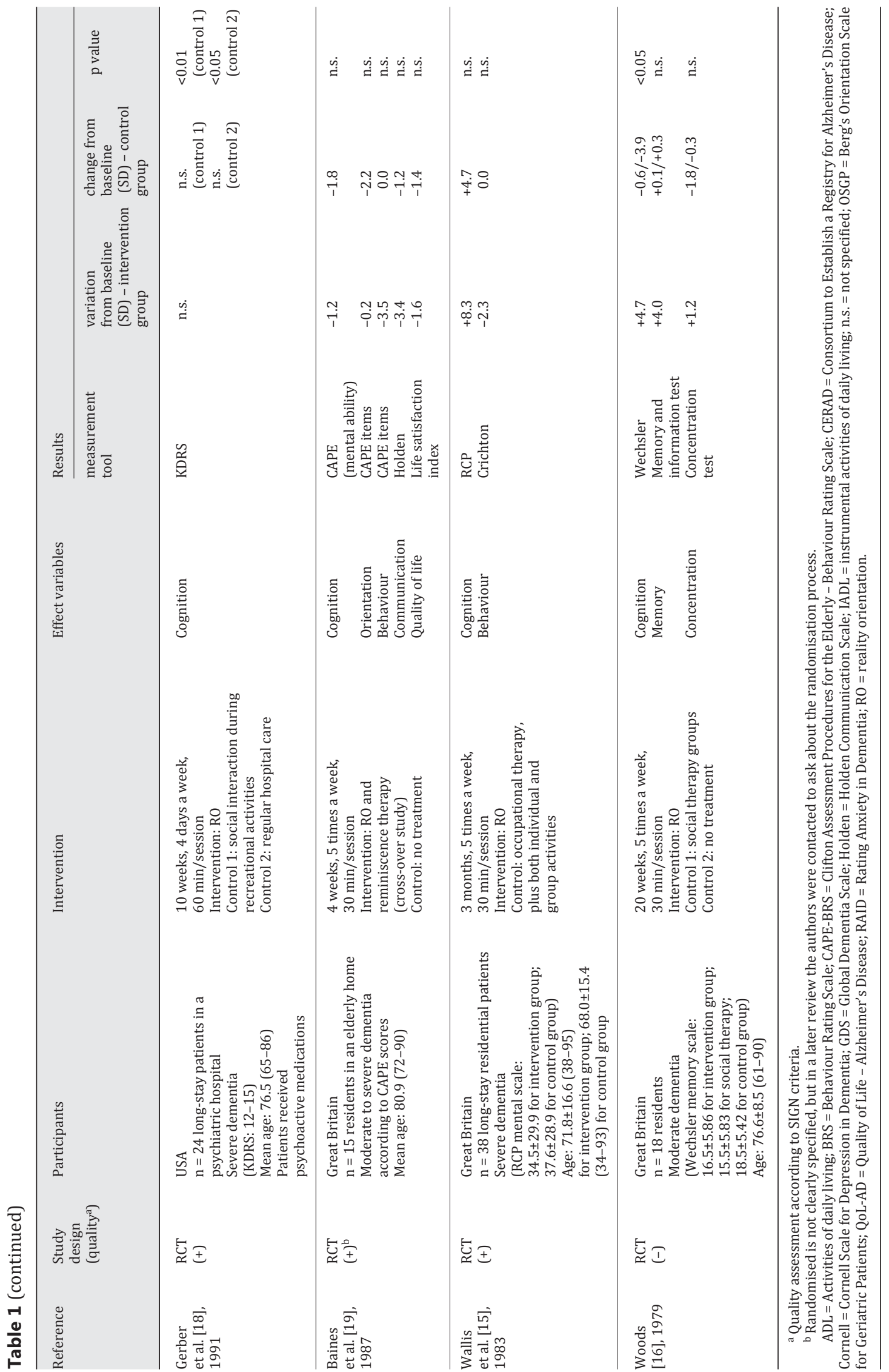


Dementia

and Geriatric
Cognitive Disorders
Dement Geriatr Cogn Disord 2013;36:363-375

DOI: $10.1159 / 000354365$

Carrion et al.: Cognitive Psychosocial Intervention in Dementia: A Systematic Review

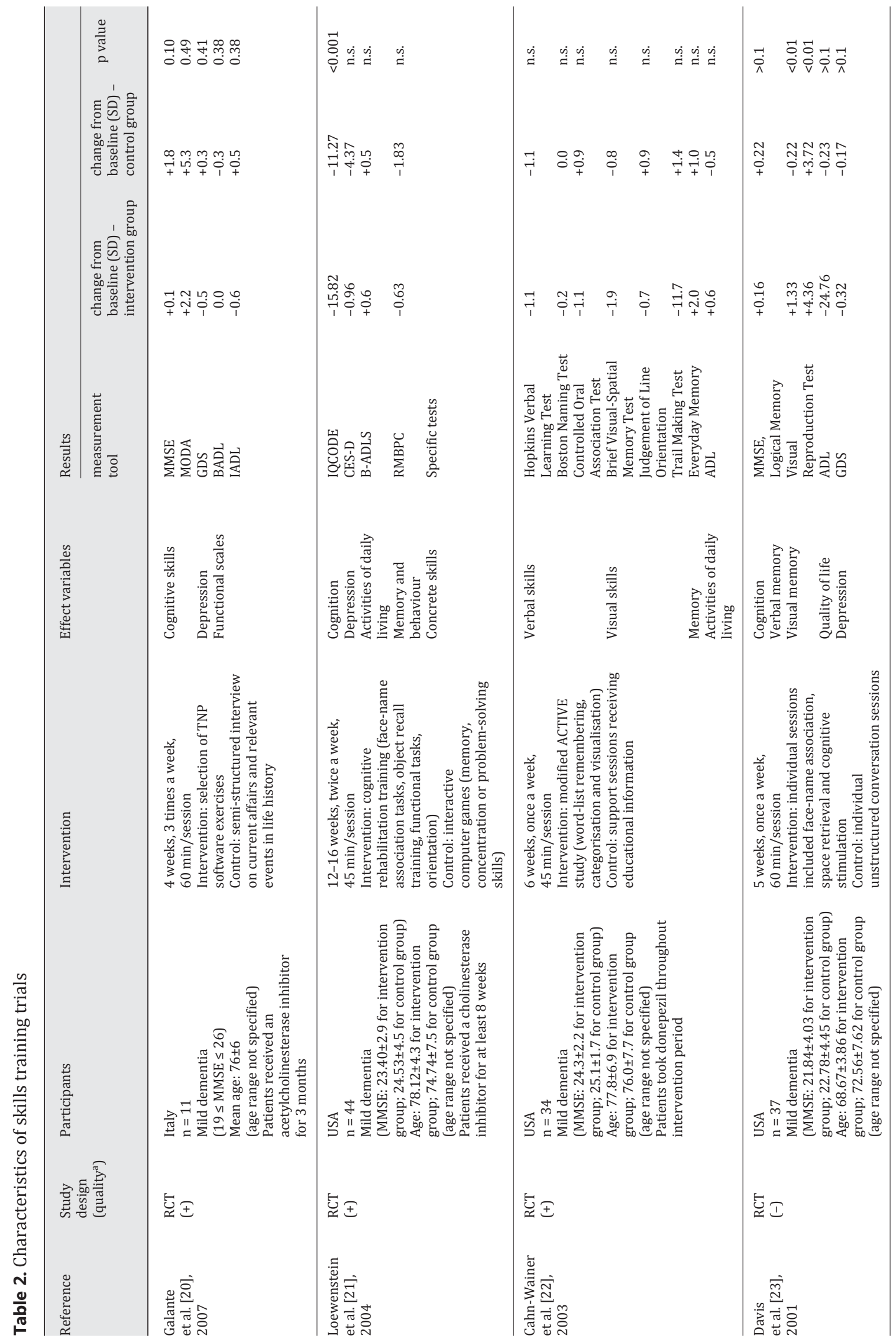


Dementia

and Geriatric
Dement Geriatr Cogn Disord 2013;36:363-375

DOI: $10.1159 / 000354365$

Carrion et al.: Cognitive Psychosocial Intervention in Dementia: A Systematic Review

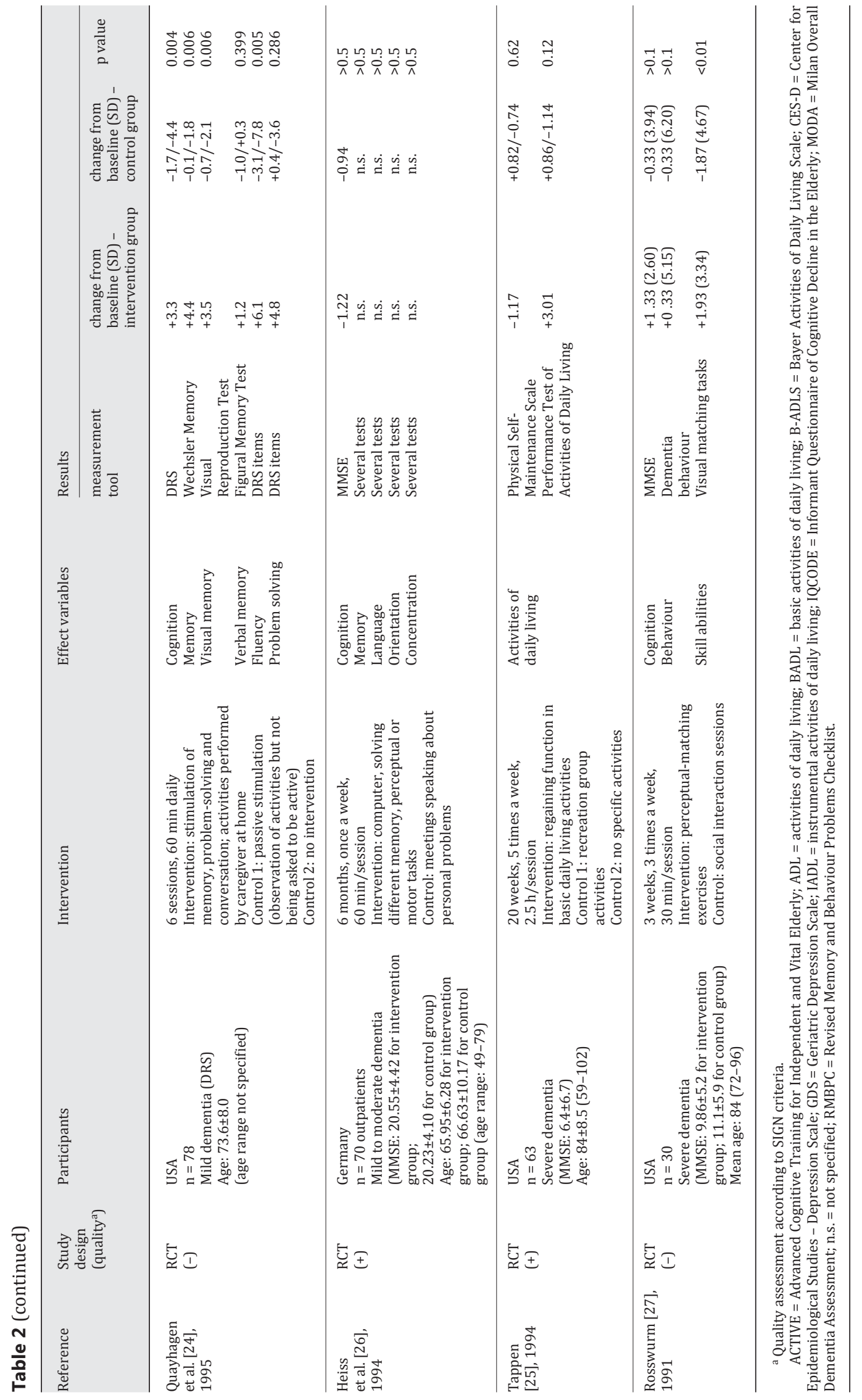


We selected 9 published trials according to the inclusion criteria [11-19]. Patients had different levels of dementia from moderate $[11-14,16,17]$ to severe $[15,18,19]$, measured using different tools such as the Mini-Mental State Examination (MMSE) [11-14, 16, 17], the Kingston Dementia Rating Scale (KDRS) [17], the Clifton Assessment Procedures for the Elderly - Cognitive Assessment Scale (CAPE-CAS) [19], the Royal College of Physicians (RCP) mental scale [15] or the Wechsler Memory Scale [16]. The number of individuals included in each study was heterogeneous, ranging from 15 [19] to 201 [11], and groups were not matched by level of impairment. Most of the trials included patients aged over 75 years, except for the trial by Woods [16], where all participants were over 60 years, and that conducted by Wallis et al. [15], where the average age was $71.8 \pm 16.6$ years for the intervention group and $68.0 \pm 15.4$ years for the control group. This study also included younger people, and the age range was wider than in the rest of the selected studies. Age ranges for all studies are shown in table 1. Patients were recruited from day centres and residential homes. In all trials, reality orientation was applied as a group therapy, except in the study by Onder et al. [13], where caregivers were trained to give reality orientation treatment at home. There was considerable heterogeneity in both the number and length of sessions. Spector et al. [11,12] used 45-min sessions twice a week for 7 weeks, while other trials used 60-min sessions two [17], three [14] or even four [18] times a week. Three trials used shorter (30-min) sessions, but five times a week [15, 16, 19]. Finally, the treatment used by Onder et al. [13] consisted of 30-min sessions three times a week at home. The duration of the intervention varied across trials, with the total duration ranging from 4 to 25 weeks. Control group interventions also differed according to the trial. Usual activities were considered as the control group in 3 of the trials $[11,12,14]$, while training in social skills both individually $[15,18]$ or working in groups $[16]$ was taken as control group intervention in some other trials, and no intervention at all in the rest of the trials $[13,16,17,19]$.

Outcomes of reality orientation interventions were analysed differently according to the trial designs. Cognitive function was tested in all trials by using the above-mentioned tools for dementia diagnosis. Regarding results, although all trials found better cognitive function in the intervention groups compared to the control groups, only 6 out of 9 found a statistically significant improvement and 2 of them had a high risk of bias (fig. 2).

Gerber et al. [18] compared reality orientation with no intervention, but also with social activities, and they reported no statistically significant differences in terms of KDRS scores between the reality orientation and the social activity groups, whereas both groups showed a clear cognitive improvement when compared to a control group. This improvement disappeared almost completely once the intervention finished. Woods [16] also used two different controlgroups (social activity and a normal intervention group), and a significantimprovement was obtained in the reality orientation group compared with both control groups. In the trial by Wallis et al. [15], a certain improvement was also found, although it was not statistically significant. When focusing on more specific cognitive function features such as memory skills, 3 of the trials found significant improvements [14,16,17]. Communication skills showed no improvement in 2 of the trials where they were analysed [12,17], although Gerber et al. [18] did find some improvement based on KDRS language subscales. In addition, orientation skills improved in 4 of the trials $[14,18,19]$.

Besides cognitive function, certain other aspects were also analysed. In 2 of the trials [12, 14], depression improved, whereas in a later trial by Spector et al. [11], no improvement was found. No other positive effect was observed when analysing anxiety, behavioural skills or health-related quality of life. 


\section{Skills Training Interventions}

Several skills training programmes were tested, which are aimed at helping patients to improve cognitive functioning, albeit only temporarily, in an attempt to stop or slow cognitive decline. Different strategies are used to this end, such as training exercises to match and categorise objects, other exercises to perform basic daily activities or simple software that helps patients to perform memory or perception activities.

Eight RCTs were selected in accordance with the inclusion criteria [20-27]. In 6 of these trials, the patients were suffering from mild dementia [20-25] and only 1 trial also included patients with moderate dementia [25]. Patients included in the other 2 trials were suffering from a severe degree of cognitive impairment [26,27]. In 7 out of the 8 studies, the degree of dementia was determined using the MMSE. The remaining trial [24] used the Mattis Dementia Rating Scale (DRS). The average age of the patients included ranged from 68 to 84 years. All trials had small samples, and the number of people included ranged from 11 [20] to 78 [24]; they were not matched by level of impairment. Three of the trials included only patients taking cholinesterase inhibitor drugs [20-22], the efficacy of which in delaying cognitive impairment stabilisation has been demonstrated for patients with mild to moderate cognitive decline [28].

Regarding the characteristics of the interventions, these were diverse, both in duration and in the selection of tools used to stimulate cognitive skills. One of the trials was based on computer activities [20], namely, the Training Neuro-Psychological (TNP) software, and another one [21] used a mixture of activities, some of which were computer cognitive training activities. Interventions in the rest of the trials consisted of organising stimulus items into meaningful categories, organising ideas and details for remembering everyday text-based information [24, 26], visualising and associating items to be remembered [22, 23, 27], lists of words to be remembered [22], using an agenda and a calendar and training in daily living activities [24, 25]. The number of sessions varied from 1 to 6 per week, and session lengths varied from $20 \mathrm{~min}$ to $2.5 \mathrm{~h}$. The duration of the studies was also very heterogeneous, ranging from 3 weeks to 6 months. Two types of control group interventions were considered: an alternative social intervention or no intervention. Effects of the intervention were mostly assessed by evaluating the patients' cognitive skills. Only 1 study also looked at depression [23]. When evaluating cognitive skills, most of the trials assessed only very specific aspects such as those related to visual memory, verbal memory or non-verbal memory.

Table 2 describes all tests used. All but 1 trial, which focused specifically on daily living activities, measured cognition as a main outcome and, although positive effects were shown in most of them, only 2 trials achieved statistically significant improvement, with only 1 being of sufficient methodological quality (fig. 2).

Patients showed modest improvement in tasks in which they were trained, but not in others. This improvement did not persist after the training intervention ended. In the trial by Heiss et al. [26], no improvement was observed when analysing cognitive intervention, but a mild improvement was observed when combining cognitive training with pharmacological therapy. None of the trials compared cognitive intervention with pharmacological therapy alone.

\section{Discussion}

Psychological interventions such as reality orientation or skills training programmes have been widely used to treat dementia. Despite being first described some time ago, their effects remain open to question as none of the studies we analysed were rated as being of high methodological quality. In fact, 35\% (6 out of 17 RCTs) were rated as being of poor methodological quality, that is, having a high risk of bias. 
Our research results suggest that reality orientation is effective in slightly slowing down functional impairment in people suffering from dementia (fig. 2) when measuring overall cognition by tools such as the MMSE or Alzheimer's Disease Assessment Scale - Cognition (ADAS-Cog), bearing in mind that slowing functional impairment does not mean slowing disease progression. However, studies are too heterogeneous to identify which intervention might be more suitable for mild, moderate or severe levels of dementia.

Although there is a possibility that the intervention itself may be teaching patients to answer cognition tests, this is something that may affect all cognition-oriented therapies, and our review shows that some of these therapies (reality orientation) are able to improve cognition and some are not. As a matter of fact, skills training studies failed to demonstrate cognitive function improvement since only 1 out the 5 good-quality trials found statistically significant cognition improvement (fig. 2).

In addition, data from several studies showed that improvements tended to be unstable and disappeared sometime after the intervention had finished. Thus, regarding policy implications, if consistent in future studies, it should be taken into account for cognitive-oriented intervention that therapy must be ongoing if it is to have long-lasting benefits.

A meta-analysis [29] on the efficacy of reality orientation in individuals suffering from dementia, which included 6 RCTs, concluded that this intervention has a positive effect on both cognitive and behavioural aspects. In the present review, we identified 9 reality orientation RCTs, including all those trials identified for the meta-analysis by Spector et al. [29]. All trials described cognitive improvements (as measured by the MMSE or similar tests) independently of sample size, which varied from 15 resident patients in a home of elderly people [19] to 197 patients assisted in different centres [11]. The length and frequency of reality orientation sessions were also diverse, ranging from 30-min sessions five times a week, to 45-min sessions twice a week.

More recently, a new meta-analysis [30] of cognitive stimulation in patients with dementia identified 15 RCTs and concluded that cognitive rehabilitation programmes benefit cognition in people suffering from dementia; however, these RCTs included several cognitive therapies, and control group interventions varied from trial to trial.

From our point of view, the heterogeneity amongst the trials prevents a meta-analysis. As a matter of fact, since our study was based on trials where the randomisation process was not always clearly explained, and since tools used to evaluate cognitive skills were different, we considered a meta-analysis to be inappropriate for this review.

Contrary to drug trials, in reality orientation and skills training interventions, both being psychological interventions, it is impossible to completely blind patients and staff to treatment. Patients may be aware that they are being treated differently, and researchers and staff may also have different expectations of groups. Moreover, although all trials selected specified that patients were randomly assigned to control or treatment groups, the randomisation process was not detailed. Two of the trials did not mention randomisation, but the randomisation process was later ascertained by contacting the authors in the course of other reviews. We therefore acknowledge that the quality of studies was suboptimal as a limitation that should be borne in mind when analysing the results of this review.

We conclude that stimulation of cognitive functions, especially by means of reality orientation, improve overall cognitive function (measured by the MMSE or ADAS-Cog) in patients suffering from dementia. Although the reviewed papers included patients with Alzheimer's or probable Alzheimer's disease, stimulation of cognitive functions may apply to dementia in general. Higher-quality trials are warranted in order to confirm these findings. Multicentre and large-sample trials may improve evidence regarding the effects of cognitive interventions on patients suffering from dementia. 


\section{Acknowledgements}

This project was supported by the Fondo de Investigación Sanitaria, Ministry of Science and Innovation, Spain (project grant ref PI09/90931).

\section{Disclosure Statement}

The authors have no conflicts of interest to declare.

\section{References}

1 Bierman EJ, Comijs HC, Jonker C, Beekman AT: Symptoms of anxiety and depression in the course of cognitive decline. Dement Geriatr Cogn Disord 2007;24:213-219.

2 Wimo A, Winblad B, Aguero-Torres H, von Strauss E: The magnitude of dementia occurrence in the world. Alzheimer Dis Assoc Disord 2003;17:63-67.

3 Aalten P, Verhey FRJ, Boziki M, Brugnolo A, Bullock R, Byrne EJ, et al: Consistency of neuropsychiatric syndromes across dementias: results from the European Alzheimer Disease Consortium. Part II. Dement Geriatr Cogn Disord 2008;25:1-8.

4 Brookmeyer F, Johnson E, Ziegler-Graham K, Arrighi HM: Forecasting the global burden of Alzheimer's disease. Alzheimers Dement 2007;3:186-191.

5 Graham JE, Mitnitski AB, Mogilner AJ, Gauvreau D, Rockwood K: Symptoms and signs in dementia synergy and antagonism. Dement Geriatr Cogn Disord 1996;7:331-335.

- 6 Aymerich M, Guillamón I, Jovell AJ: Health-related quality of life assessment in people with multiple sclerosis and their family caregivers. A multicenter study in Catalonia (Southern Europe). Patient Prefer Adherence 2009;3:311-321.

7 Markowitz JS, Gutterman EM, Sadik K, Papadopoulos G: Health-related quality of life for caregivers of patients with Alzheimer disease. Alzheimer Dis Assoc Disord 2003;17:209-214.

8 American Psychiatric Association: Practice guideline for the treatment of patients with Alzheimer's disease and other dementias. Arlington, American Psychiatric Association, 2007.

9 Scottish Intecollegiate Guidelines Network (SIGN): SIGN 50: a guideline developer's handbook. http://www. sign.ac.uk/guidelines/fulltext/50/index.html. Edinburgh, Scottish Intercollegiate Guidelines Network, 2008.

10 Taulbee LR, Folsom JC: Reality orientation for geriatric patients. Hosp Community Psychiatry 1966;17:133135.

11 Spector A, Thorgrimsen L, Woods B, Royan L, Davies S, Butterworth M, et al: Efficacy of an evidence-based cognitive stimulation therapy programme for people with dementia: randomised controlled trial. $\mathrm{Br} \mathrm{J}$ Psychiatry 2003;183:248-254.

12 Spector A, Orrell M, Davies S, Woods B: Can reality orientation be rehabilitated? Development and piloting of an evidence-based programme of cognition-based therapies for people with dementia. Neuropsychol Rehabil 2001;11:377-397.

13 Onder G, Zanetti O, Giacobini E, Frisoni GB, Bartorelli L, Carbone G, et al: Reality orientation therapy combined with cholinesterase inhibitors in Alzheimer's disease: randomised controlled trial. Br J Psychiatry 2005;187: 450-455.

14 Baldelli MV, Pirani A, Motta M, Abati E, Mariani E, Manzi V: Effects of reality orientation therapy on elderly patients in the community. Arch Gerontol Geriatr 1993;17:211-218.

15 Wallis GG, Baldwin M, Higginbotham P: Reality orientation therapy - a controlled trial. Br J Med Psychol 1983; 56:271-277.

16 Woods RT: Reality orientation and staff attention: a controlled study. Br J Psychiatry 1979;134:502-507.

17 Breuil V, de Rotrou J, Forette F, Tortrat D, Ganansia-Ganem A, Frambourt A, et al: Cognitive stimulation of patients with dementia: preliminary results. Int J Geriatr Psychiatry 1994;9:211-217.

18 Gerber GJ, Prince PN, Snider HG, Atchison K, Dubois L, Kilgour JA: Group activity and cognitive improvement among patients with Alzheimer's disease. Hosp Community Psychiatry 1991;42:843-845.

$>19$ Baines S, Saxby P, Ehlert K: Reality orientation and reminiscence therapy. A controlled cross-over study of elderly confused people. Br J Psychiatry 1987;151:222-231.

20 Galante E, Venturini G, Fiaccadori C: Computer-based cognitive intervention for dementia: preliminary results of a randomized clinical trial. G Ital Med Lav Ergon 2007;29(suppl 3):B26-B32.

-21 Loewenstein DA, Acevedo A, Czaja SJ, Duara R: Cognitive rehabilitation of mildly impaired Alzheimer disease patients on cholinesterase inhibitors. Am J Geriatr Psychiatry 2004;12:395-402.

22 Cahn-Weiner DA, Malloy PF, Rebok GW, Ott BR: Results of a randomized placebo-controlled study of memory training for mildly impaired Alzheimer's disease patients. Appl Neuropsychol 2003;10:215-223.

23 Davis RN, Massman PJ, Doody RS: Cognitive intervention in Alzheimer disease: a randomized placebocontrolled study. Alzheimer Dis Assoc Disord 2001;15:1-9. 
24 Quayhagen MP, Quayhagen M, Corbeil RR, Roth PA, Rodgers JA: A dyadic remediation program for care recipients with dementia. Nurs Res 1995;44:153-159.

25 Tappen RM: The effect of skill training on functional abilities of nursing home residents with dementia. Res Nurs Health 1994;17:159-165.

-26 Heiss WD, Kessler J, Mielke R, Szelies B, Herholz K: Long-term effects of phosphatidylserine, pyritinol, and cognitive training in Alzheimer's disease. A neuropsychological, EEG, and PET investigation. Dementia 1994; 5:88-98.

27 Rosswurm MA: Attention-focusing program for persons with dementia. Clin Gerontol 1991;10:3-16.

-28 Doody RS, Stevens JC, Beck C, Dubinsky RM, Kaye JA, Gwyther L, et al: Practice parameter: management of dementia (an evidence-based review). Report of the Quality Standards Subcommittee of the American Academy of Neurology. Neurology 2001;56:1154-1166.

29 Spector A, Davies S, Woods B, Orrell M: Reality orientation for dementia: a systematic review of the evidence of effectiveness from randomized controlled trials. Gerontologist 2000;40:206-212.

30 Woods B, Aguirre E, Spector A, Orrell M: Cognitive stimulation to improve cognitive functioning in people with dementia. Cochrane Database Syst Rev 2012:CD005562. 